\title{
Aprendizado Evolucionário, Inércia Inflacionária e Recessão em Desinflações Monetárias*
}

\author{
Marco Bonomo** \\ Vinícius Carrasco ${ }^{* * *}$ \\ Humberto Moreira ${ }^{* * * *}$
}

Sumário: 1. Introdução; 2. Inércia inflacionária e aprendizado evolucionário: credibilidade perfeita e desinflação imediata; 3. Conclusão.

Palavras-chave: custos de desinflação monetária; teoria dos jogos evolucionários; dinâmica replicadora; inércia inflacionária.

Códigos JEL: E31; E52.

Este artigo busca justificar a evidência casual de que desinflações monetárias tendem a ser acompanhadas de queda no produto utilizando-se do arcabouço da teoria dos jogos evolucionários. A aplicação deste arcabouço tem o seu apelo porque combina duas hipóteses freqüentemente apontadas como responsáveis pelo fato de que desinflações críveis têm custos: racionalidade limitada e falha de coordenação. A análise das desinflações constitui-se na análise da transição entre dois equilíbrios estacionários. A dinâmica da economia em direção ao novo equilíbrio do jogo de estabelecimento de preços é dada pela dinâmica replicadora, que usa uma regra de seleção de estratégias simples e intuitiva: estratégias que apresentam desempenho pior que a média têm sua utilização reduzida ao longo do tempo. O custo da desinflação em nosso modelo depende do quão rápido agentes passivos, que continuam a adotar a estratégia que era ótima para o equilíbrio inflacionário, se convertem em agentes ativos, que adotam estratégias maximizadoras durante a transição.

This paper aims at explaining the casual evidence that monetary disinflations produce recessions, using the evolutionary game theory framework. This is an appealing framework for analyzing this

\footnotetext{
*Artigo Recebido em nov. 2000 e aprovado em jan. 2003. Este artigo é uma versão modificada da dissertação de mestrado de Vinícius Carrasco e contou com o apoio da CAPES e CNPq. Gostaríamos de agradecer aos comentários e sugestões feitas por Heitor Almeida, Ilan Goldfajn e dois pareceristas anônimos.

${ }^{* *}$ EPGE/FGV

${ }^{* * *}$ Stanford University

**** EPGE/FGV
} 
issue because it combines two ingredients commonly blamed for the costs of credible disinflations: limited rationality and coordination failures. The study of a disinflation episode is the analysis of a transition between two steady state equilibria. The dynamics between those two equilibria is generated by the replicator dynamics, which is based on an appealing strategy selection rule: the use of strategies that have a worse than average performance is reduced through time. The disinflation costs in our model depend on how fast passive agents - who continue to use the strategy that was optimal in the inflationary equilibrium - are converted into active agents - who adopt maximizing strategies during the transition.

\section{Introdução}

Há uma clara evidência histórica de que tentativas de combate à inflação baseadas em controle de demanda agregada resultam em quedas de produto. Isto decorre da redução defasada no ritmo de crescimento de preços, o que faz com que a recessão aconteça antes que a redução desejada na inflação se efetive.

Há uma vasta literatura que enfatiza que o custo das desinflações deve-se à rigidez nominal nominal de preços, que seria uma característica essencial das economias monetárias. Acreditava-se que a hipótese de rigidez nominal associada à intercalação dos ajustes de preços por si só justificariam o caráter recessivo das desinflações monetárias. Tal crença baseava-se no suposto de que modelos que geram inércia de preços também gerariam inércia inflacionária. Como mostrado por Ball (1994), tal presunção era infundada.

As hipóteses de rigidez nominal e de contratos intercalados podem ser interpretadas como sendo um artifício de modelagem para captar o problema de coordenação, que desde Tobin acreditava-se ser o cerne do problema das desinflações monetárias. Simonsen (1983) resolve atacar diretamente a questão modelando explicitamente as decisões individuais de determinação de preços como um jogo de estabelecimento de preços entre as firmas. Simonsen (1983) critica a relevância do conceito de equilíbrio de Nash (E.N.) para a solução deste jogo, adotando uma solução em que os agentes agem com um grau extremo de prudência. Esta idéia foi aperfeiçada em Dow et alii (1993), que se utilizam do conceito de incerteza knightiana em jogos introduzido por Dow e Werlang (1994).

Neste artigo, trataremos o problema da inércia inflacionária como sendo um problema de coordenação envolvendo racionalidade limitada por parte dos agentes. Mais especificamente, suporemos que parte dos agentes não é completamente racional, mas tem um aprendizado do tipo preconizado em jogos evolucionários. 
Isto afeta o resultado macroeconômico direta e indiretamente. O efeito indireto deve-se à complementaridade estratégica no estabelecimento de preços que faz com que o estabelecimento de preços dos agentes racionais seja influenciada pelos preços dos agentes passivos. Como conseqüência uma pequena proporção de agentes evolucionários é suficiente para gerar inércia inflacionária e perda de produto mesmo para estabilizações perfeitamente críveis. Este resultado confirma a conjectura de Ball (1991) e reflete neste modelo específico a proposição mais geral derivada por Bonomo (1992): em uma situação de complementaridade estratégica alta, uma pequena proporção de agentes não-neutros é capaz de causar substancial não-neutralidade na economia.

A hipótese da existência de um grupo de agentes não-racionais em modelos de dinâmica inflacionária tem sido utilizada de diversas formas em artigos recentes para justificar a inércia inflacionária. Roberts (1997) justifica a inércia inflacionária a partir de expectativas que têm um componente adaptativo. Ball (2000) refina a idéia de expectativa adaptativa ao propor que agentes façam previsões ótimas com base na série de inflações passada, ignorando outras variáveis. Ele argumenta que a perda incorrida pela quase-racionalidade é pequena no caso da economia americana. Amato e Laubach (2003) propõem um modelo no qual uma fração dos agentes usam regras de bolso: firmas determinam o preço extrapolando a inflação passada e consumidores escolhem consumir uma fração da renda disponível.

Acreditamos que a inovação deste trabalho esteja em propor uma dinâmica para o desvio da racionalidade que interage com a transição entre estados inflacionários. Lucas (1986) considera que as regras de decisão associadas à racionalidade (mais especificamente, à expectativas racionais) sejam estados estacionários de algum processo adaptativo. Uma idéia similar motiva este trabalho. Quando parte dos agentes reajusta os seus preços automaticamente de acordo com a inflação corrente durante o estado estacionário, estão agindo otimamente, embora por inércia. Quando a política de desinflação é adotada, a inércia se torna custosa e este custo determina a velocidade de conversão dos agentes passivos em racionais. De forma mais direta, tomaremos emprestado da teoria dos jogos evolucionários a chamada dinâmica replicadora, que estabelece que a proporção de agentes adotando uma certa estratégia aumenta (diminui) se tal estratégia gera uma payoff maior (menor) que o payoff médio das estratégias. A taxa de conversão dos agentes é crescente na perda de lucro causada pelo comportamento passivo. No nosso contexto, os agentes passivos obterão um payoff menor que o da média da economia na transição e, portanto, tenderão a desaparecer assintoticamente, o que implicará na convergência da economia para o equilíbrio em expectativas racionais 
no longo prazo (isto é, no longo prazo, todos os jogadores terão localizado o novo E.N.). A dinâmica deste aprendizado interage com a trajetória de desinflação da política monetária, determinando a inércia inflacionária e a perda do produto.

Alguns resultados gerados pelo nosso modelo são mais realistas do que os obtidos em modelos de rigidez nominal. Em particular, ele gera inércia inflacionária e perda de produto substanciais, independentemente do grau de credibilidade da desinflação, o que contrasta com a incapacidade de modelos de rigidez nominal de gerar estes mesmos resultados sob credibilidade perfeita. ${ }^{1}$ Em outros aspectos nosso modelo têm implicações similares aos modelos com rigidez nominal, como a menor persistência da recessão gerada em desinflações a partir de inflações altas. ${ }^{2}$

A segunda seção do artigo apresenta, a partir da regra ótima de estabelecimento de preços resultante de uma economia em competição monopolística, como a proposta por Blanchard e Kiyotaki (1987), o jogo de estabelecimento de preços que exploraremos. Desenvolveremos o exercício de estabilização de preços com credibilidade perfeita sob a hipótese de que parte dos agentes agem de forma passiva. Definiremos a dinâmica replicadora para estes agentes, obtendo, assim, uma trajetória no tempo para os mesmos. Derivaremos as trajetórias do nível geral de preços e do produto sob esta hipótese, comparando com o resultado sob expectativas racionais. A última seção tece os comentários finais.

\section{Inércia Inflacionária e Aprendizado Evolucionário: Credibilidade Perfeita e Desinflação Imediata}

Nesta seção, apresentamos o resultado de uma tentativa de estabilização de preços em uma economia em que parte dos agentes têm inicialmente estratégia passiva e aprende apenas evolucionariamente o novo equilíbrio. Faremos a suposição de que o processo de aprendizado destes agentes segue uma regra bastante simples: estratégias que geram payoff maior do que o payoff médio da economia tendem a se reproduzir. Mostraremos que este processo de aprendizagem resulta, no longo prazo, em um equilíbrio em expectativas racionais. No entanto, como não se atinge este equilíbrio instantaneamente, veremos que a economia sofrerá uma perda de produto decorrente da inércia inflacionária.

\footnotetext{
${ }^{1}$ Isto se aplica tanto a modelos com regras dependentes do tempo (Ball, 1994), como para modelos com regras dependentes do estado (Almeida e Bonomo, 2002).

${ }^{2}$ Este resultado também foi obtido por Bonomo e Carvalho (2003) para um modelo com ridigez nominal e regras dependentes do tempo.
} 


\subsection{O equilíbrio estacionário com inflação}

Considerando uma economia com competição monopolística entre produtores, com produtos com elasticidade substituição constante, e moeda na função utilidade, como a descrita pelo modelo de Blanchard e Kiyotaki (1987), pode-se mostrar que o preço relativo ótimo estabelecido pelo produtor $i$ é

$$
P_{i}=\alpha P^{a} M^{1-a}
$$

onde $P$ é uma medida do nível geral de preços (que consideraremos como sendo igual à média geométrica dos preços adotados pelos agentes), $M$ é o estoque nominal de moeda e $0<a<1$ é um parâmetros que depende de alguns parâmetros da função utilidade do produtor-consumidor.

A equação (1) reflete a interdependência estratégica no estabelecimento de preços nesta economia. O produtor-consumidor $i$, ao estabelecer o seu preço ótimo, leva em consideração, através do nível geral de preços $P$, os preços escolhidos pelos demais produtores. O E.N. simétrico para este jogo de estabelecimento de preços é dado pela condição

$$
P_{i}=P=\alpha_{0} M
$$

onde $\alpha_{0}=\alpha^{\frac{1}{1-a}}$. Portanto, o preço ótimo de cada produtor (e o nível geral preços) é uma função linear do estoque nominal de moeda da economia.

É razoável supor que, em um ambiente de inflação crônica, o estoque nominal de moeda cresça a uma taxa constante, $\pi$, isto é,

$$
M(t)=M_{0} \exp (\pi t)
$$

Substituindo-se (3) em (2), tem-se, prontamente,

$$
P_{i}=P=\alpha_{0} M_{0} \exp (\pi t)
$$

Claramente, em uma situação de equilíbrio, o nível geral de preços cresce à mesma taxa, $\pi$, que o estoque nominal de moeda.

\subsection{Estratégias de determinação de preços durante a estabilização}

Suponhamos que a autoridade monetária anuncie (de forma totalmente crível) que a partir de um instante $t^{*}$ do tempo o estoque nominal de moeda se estabilize em $M_{0} \exp \left(\pi t^{*}\right)$. Em outras palavras, que a trajetória do estoque nominal de moeda seja descrita por 


$$
M(t)=M_{0} \exp \left(\pi t^{*}\right), \forall t \geq t^{*}
$$

Em um equilíbrio de expectativas racionais, dada a total credibilidade do anúncio de estabilização, os agentes adotariam imediatamente a estratégia associada ao E.N., isto é, estabeleceriam seus preços de acordo com (2'), o que implicaria

$$
P(t)=\alpha_{0} M_{0} \exp \left(\pi t^{*}\right) \equiv P_{0}, \forall t \geq t^{*}
$$

e, como conseqüência, uma taxa de inflação igual a zero a partir da efetivação da nova política monetária. Tem-se, como resultado de um anúncio crível de mudança de política monetária, a possibilidade de uma estabilização de preços sem efeito recessivo sobre a economia. ${ }^{3}$

Tal resultado depende crucialmente da hipótese de que (todos) os agentes estejam aptos a localizar o novo E.N. da economia quando da efetivação da nova política, conforme apontado por Simonsen (1983). Adotaremos a hipótese de que uma fração dos produtores continue, de forma passiva, adotando a estratégia associada à política monetária anterior, i.e., é como se parte dos agentes não percebessem que, com a mudança da política monetária, o jogo muda. Claramente, tal passividade tem um custo: na economia aqui descrita, os agentes incorrem em uma perda por estarem fora de seu preço ótimo, sendo esta perda uma função do número de produtores que ajustam seus preços de acordo com a nova política monetária. Mais especificamente, suporemos que uma proporção $k>0$ de agentes continuem estabelecendo seu preço em acordo com (2'), que pode ser reescrita para instantes de tempo maiores ou iguais a $t^{*}$ como

$$
P_{n}\left(t^{*}+t^{\prime}\right) \equiv P_{n}\left(t^{\prime}\right)=\alpha_{0} M_{0} \exp \left[\pi\left(t^{*}+t^{\prime}\right)\right] \equiv P_{0} \exp \left(\pi t^{\prime}\right)
$$

para $t^{\prime} \geq 0$ e onde $P_{0}$ corresponde ao E.N. simétrico sob nova política monetária (veja (5)).

A proporção complementar $(1-k)$ de agentes na economia adota a estratégia ótima $P_{r}\left(t^{\prime}\right)=\alpha P^{a} M^{1-a}$. Usando-se o fato de que a política monetária para instantes de tempo maiores ou iguais a $t^{*}$ é dada por $M_{0} \exp \left(\pi t^{*}\right)=\frac{P_{0}}{\alpha_{0}}$, temos que o preço ótimo é dado por

\footnotetext{
${ }^{3}$ Uma proxy para o produto real desta economia é o estoque real de moeda M/P. É óbvio, portanto, que, em Expectativas Racionais, o produto da economia não se altera quando da efetivação da nova política dado que o nível geral de preços não apresenta um comportamento de inércia.
} 


$$
P_{r}=\alpha\left[P_{r}^{1-k}\left(P_{0} \exp \left(\pi t^{\prime}\right)\right)^{k}\right]^{a}\left[\frac{P_{0}}{\alpha_{0}}\right]^{1-a}
$$

que, resolvendo para $P_{r},{ }^{4}$ implica

$$
P_{r}=P_{0} \exp \left(\gamma(k) t^{\prime}\right)
$$

onde

$$
\gamma(k)=\frac{\pi a k}{1-a(1-k)}<\pi, \forall k
$$

Note que $\gamma(0)=0$, o que implica que os agentes otimizadores adotariam a estratégia relativa ao E.N. simétrico caso não houvesse agentes passivos nesta economia. Utilizando-se a expressão para o preço ótimo e a expressão para o preço adotado pela proporção $k$ de agentes míopes, temos que o nível geral de preços pode ser descrito como

$$
P=P_{r}^{1-k}\left(P_{0} e^{\pi t^{\prime}}\right)^{k}=P_{0} \exp \left[k \pi t^{\prime}+(1-k) \gamma(k) t^{\prime}\right]
$$

O resultado de inércia decorre de dois efeitos. O primeiro deles é o efeito direto de uma proporção de agentes na economia continuar a reajustar seus preços à mesma taxa que ajustavam sob a política anterior. O efeito indireto segue do fato de que, ao estabelecer otimamente seu preço relativo, o agente racional leva em consideração, via nível geral de preços, a estratégia adotada pela proporção de agentes passivos e, como conseqüência, segue reajustando seu preço (ainda que a uma taxa menor que $\pi$ ). Em outras palavras, neste trabalho, consideramos inércia inflacionária como sendo resultado da interação entre a passividade de alguns agentes e a complementaridade estratégica existente no jogo de estabelecimento de preços, que faz com que a passividade dos agentes irracionais tenha influência sobre o preço estabelecido pelos agentes racionais.

Observe que, se todos os agentes se coordenassem prontamente para o novo equilíbrio, não teríamos o resultado de inércia. Estamos em uma situação em que haveria ganhos para todos os agentes se todos estabelecessem seus preços em $P_{0}$. No entanto, individualmente, isto só é ótimo se tal estratégia também for adotada pelos demais agentes. Em outras palavras, há um problema de falha de

\footnotetext{
${ }^{4}$ Implicitamente, estamos usando o fato de que os agentes racionais conhecem a estratégia utilizada pelos agentes míopes, o que os capacita a inferir, a partir do nível geral de preços, a proporção de agente irracionais. Trata-se da conhecida hipótese de que as estratégias a disposição dos agentes são de conhecimento comum.
} 
coordenação. No nosso modelo, é a existência de agentes míopes que impede que os agentes se coordenem na estratégia $P_{0}$.

Cooper e John (1988) é uma referência seminal na constatação de que resultados Keynesianos podem ser decorrentes de falhas de coordenação dos agentes em situações que apresentam complementaridade estratégica. A hipótese de que os custos de desinflação podem ser explicados por alguma forma de comportamento que envolva desvios em relação à racionalidade já foi levantada por Ball (1991). Outro artigo que serve de motivação para este trabalho é o de Roberts (1997). Neste trabalho, os dados americanos de inflação são condizentes com um modelo em que os preços são rígidos e as expectativas são uma combinação convexa entre expectativas racionais e backward looking. O intuito básico deste trabalho é propor um mecanismo de aprendizado para os agentes míopes que consiga descrever sua trajetória ao longo do tempo. Faremos isto através da dinâmica replicadora. Com a trajetória destes agentes em mãos, conseguiremos descrever a trajetória do nível geral de preços e do produto desta economia.

\subsection{Dinâmica replicadora como mecanismo de seleção de estra- tégias}

Adotaremos a hipótese de que os agentes míopes incorrem em uma perda quadrática ao não estabelecerem otimamente seus preços (obviamente, os agentes racionais, por adotarem o preço ótimo, não incorrem em perda alguma). Mais especificamente, suporemos que a perda toma a seguinte forma

$$
L_{n}=-\beta\left[P_{n}-P_{r}\right]^{2}
$$

onde $\beta>0$.

Podemos justificar tal perda através de uma aproximação de segunda ordem da função lucro ao redor do preço ótimo. Vejamos:

$$
\pi\left(P_{i}\right) \cong \pi\left(P^{*}\right)+\pi^{\prime}\left(P^{*}\right)\left[P_{i}-P^{*}\right]+\frac{1}{2} \pi^{\prime \prime}\left(P^{*}\right)\left[P_{i}-P^{*}\right]^{2}
$$

A condição de primeira ordem para lucro máximo é $\pi^{\prime}\left(P^{*}\right)=0$ e a condição de segunda ordem é $\pi "\left(P^{*}\right) \leq 0 .{ }^{5}$ Suporemos que a desigualdade vigore estritamente. Portanto, podemos aproximar a função perda por (10). Substituindo-se as expressões para o preço estabelecido pelos agentes não racionais (6) e para o preço ótimo (7), temos que a perda pode ser descrita por

\footnotetext{
${ }^{5}$ Note-se que o regime de competição aqui é de competição monopolística e, portanto, a hipótese de que a função lucro é (ao menos localmente) côncava não é inadequada.
} 


$$
L_{n}=-\beta P_{0}^{2}\left[\exp \left(\pi t^{\prime}\right)-\exp \left[\frac{\pi a k t^{\prime}}{1-a(1-k)}\right]\right]^{2}
$$

Tomaremos emprestado da teoria dos jogos evolucionários a chamada dinâmica replicadora, que estabelece que a proporção de jogadores adotando determinada estratégia aumenta (diminui) se o payoff obtido por esta estratégia for maior (menor) que o payoff médio. Mais especificamente, a dinâmica replicadora toma a seguinte forma (veja Weibull (1996) ou Fudenberg e Levine (1998)):

$$
\frac{d x_{i}}{d t}(t)=x_{i}(t)\left[U\left(a_{i}, x(t)\right)-\bar{U}(x(t))\right], \quad i=1,2, \ldots ., n,
$$

no qual $x_{i}$ é a proporção de jogadores adotando a estratégia $a_{i}, U\left(a_{i}, x(t)\right)$ é o payoff obtido pela estratégia $a_{i}$ e $\bar{U}(x(t))$ é o payoff médio. Esta regra de aprendizado simples gera resultados bastante fortes como: (i) se um perfil de estratégias mistas $x^{*}=\left(x_{1}^{*}, x_{2}^{*}, \ldots, x_{I}^{*}\right)$ é um E.N. simétrico, então $x^{*}$ é um estado estacionário do sistema (11); (ii) se o estado estacionário $x^{*}$ é estável, então $\left(x_{1}^{*}, x_{2}^{*}, \ldots, x_{I}^{*}\right)$ é um E.N.; e (iii) se uma estratégia pura $a_{i}$ for estritamente (ou iteradamente) dominada, então a proporção de agentes que a adota converge para 0 no longo prazo. $^{6}$

No nosso caso específico, temos duas estratégias: os agentes ajustam otimamente seus preços ou continuam adotando o preço associado à política monetária anterior. O resultado (iii) torna equivalente a nossa abordagem com duas estratégias ao caso em que há um número finito de estratégias sendo todas, com exceção de uma: a de adotar o preço ótimo, iteradamente dominadas. No equilíbrio inflacionário as duas estratégias geram payoffs equivalentes, já que a estratégia passiva é também ótima nesta situação. Quando muda a política monetária a situação muda, e os que utilizam a estratégia ativa continuam sendo otimizadores. Os segundos incorrem na perda expressa por (10'). Utilizando-se as expressões para a dinâmica replicadora e o payoff médio da economia, temos que a dinâmica para a proporção de agentes que não estabelecem seus preços otimamente é dada por (claramente, o payoff médio da economia é dado por $k L_{n}$ ).

$$
\frac{d k}{d t}=k(1-k) L_{n}
$$

que, substituindo-se a expressão para $L_{n}$, toma a seguinte forma

\footnotetext{
${ }^{6}$ Esta última condição vale para qualquer condição inicial que seja ponto interior do simplex de estratégias. Para as provas de tais resultados, remetemos o leitor a Weibull (1996).
} 


$$
\frac{d k}{d t}(t)=-k(1-k) \beta P_{0}^{2}\left(\exp \left(\pi t^{\prime}\right)-\exp \left[\frac{\pi a k t^{\prime}}{1-a(1-k)}\right]\right)^{2}
$$

Há dois estados estacionários para esta equação diferencial. Eles correspondem $\operatorname{aos} \operatorname{estados}^{7} k=0$ e $k=1$. Note, no entanto, que para todo $k$ diferente de 0 e 1 , tem-se $\frac{d k}{d t}<0$. Conclui-se que o estado estacionário associado a $k=0$ é globalmente estável. Não é possível encontrar uma solução analítica para (11'), o que sugere que a equação diferencial acima deve ser resolvida através de métodos numéricos. ${ }^{8}$ No entanto, uma característica da dinâmica é observável sem que tenhamos uma solução explícita. Para instantes de tempo suficientemente grandes, a proporção de agentes míopes vai a zero. Esta constatação relaciona-se diretamente com um dos resultados apresentados. Vimos que estratégias estritamente dominadas tendem a desaparecer da população para qualquer condição inicial interior, isto é, neste caso, para qualquer condição inicial em que haja alguns agentes adotando o preço ótimo. No nosso exemplo, não adotar o preço ótimo é uma estratégia estritamente dominada.

\subsection{Persistência inflacionária e perda de produto}

Com a expressão que descreve a trajetória para $k$, podemos fazer alguns exercícios relativos a persistência inflacionária e perda de produto quando de uma tentativa de estabilização de preços. Definindo, $p=\ln P$ e $p_{0}=\ln P_{0}$, temos, ao tomarmos o logarítimo da expressão do nível geral de preços (8),

$$
p=p_{0}+\pi k t^{\prime}+(1-k) \gamma(k) t^{\prime}
$$

Independente de não ser possível derivar explicitamente uma expressão para a trajetória dos agentes passivos, um resultado é aparente:

\footnotetext{
${ }^{7} \mathrm{O}$ resultado de que $k=0$ é um estado estacionário da dinâmica replicadora advém do fato que ajustar o preço otimamente é um E.N. Veja o resultado (i).

${ }^{8}$ Poderíamos linearizar (11') em torno de $k=0$ e, como conseqüência, obter uma solução analítica para a dinâmica linearizada. Deve-se notar, entretanto, que, ao linearizar (11'), estaríamos retirando todo o efeito da complementaridade estratégica sobre a trajetória dos agentes míopes. Isto faria com que os efeitos reais da existência de agentes míopes fossem diminuídos.
} 
Proposição 2.1 Suponha que a $<1$ e $k(0) \leq 1-\varepsilon$, para algum $\varepsilon>0$. Tem-se, então, que $p \rightarrow p_{0}$ quando $t^{\prime} \rightarrow \infty$.

Prova Note que $0 \leq p-p_{0} \leq \pi k t^{\prime}+\frac{\pi a k t^{\prime}}{1-a}$, de forma que é suficiente mostrar que $k t^{\prime}$ vai a zero. Com este propósito, da dinâmica replicadora e pela hipótese sobre os valores de a e $k(0)$, temos que, após algumas manipulaçôes, $\frac{d k}{d t} \leq-k \varepsilon \beta P_{0}^{2}$ $[\exp (\pi)-1]^{2}$, para todo $t^{\prime} \geq \frac{1-\varepsilon a}{1-a} \equiv t$. Tem-se, então, que $0 \leq t^{\prime} k\left(t^{\prime}\right) \leq t^{\prime} k(t) \exp$ $\left\{-\varepsilon \beta P_{0}^{2}[\exp (\pi)-1]^{2}\left(t^{\prime}-t\right)\right\}$ para todo $t^{\prime} \geq t$. Tomando o limite quando $t^{\prime}$ vai a infinito, o resultado segue.

Em palavras, sob a hipótese de a trajetória dos agentes passivos seja dada pela dinâmica (11'), temos que o nível geral de preços converge para o nível de preços associado ao equilíbrio em expectativas racionais, $P_{0}$, desde que o parâmetro que capta a interação estratégica entre os agentes, $a$, e a fração inicial de agentes passivos, $k(0)$, sejam menores que 1 . Portanto, a economia converge para uma situação de inflação zero, ou, em outras palavras, a economia converge para uma situação em que todos os agentes adotam a estratégia associada ao E.N. simétrico, que implicará um nível geral de preços constante em $P_{0}$. Entretanto, ao contrário do que ocorreria em um equilíbrio em expectativas racionais, a trajetória da economia em direção a este estado estacionário é muito mais tortuosa devido a existência de agentes passivos.

A figura 1 demonstra este fato para uma proporção inicial de agentes passivos de $5 \%$, uma taxa de inflação de $5 \%$ e para alguns valores de $a$ (estamos supondo que $\beta=1$ ). Observe que considerando uma alta complementaridade estratégica $(a=0,9)$ faz com que os efeitos reais da passividade sejam majorados. Isto ocorre por duas razões: (i) quanto maior a complementaridade estratégica, mais peso os agentes racionais dão ao comportamento dos agentes passivos, o que implica que os próprios agentes racionais ajustam mais rapidamente os seus preços, e (ii) a perda dos agentes irracionais é menor, devido ao que foi exposto no item (i), e, portanto, sua velocidade de desaparecimento é menor. 
O (logaritmo do) nível geral de preços de equilíbrio de longo-prazo é igual a $P_{0}$ (que normalizamos para zero). Entretanto, tem-se que, para um período de tempo não desprezível, esta medida assume valores maiores que o associado ao longo-prazo. ${ }^{9}$ O produto desta economia é o negativo do nível geral de preços. Tem-se, como conseqüência, que os efeitos reais da tentativa de estabilização são negativos e persistentes. Deve-se ressaltar que complementaridade estratégica é fundamental para o principal resultado do trabalho: pequenos (em termos de proporção) desvios de racionalidade podem gerar substanciais efeitos reais na economia se houver interação estratégica entre os agentes. Este resultado é um caso particular do princípio geral derivado em Bonomo (1992), segundo o qual uma pequena proporção de agentes não-neutros pode gerar importantes efeitos reais na economia se a complementariedade estratégica for suficientemente forte. Para ilustrar este resultado, a figura 1 também descreve a trajetória do nível geral de preços sem complementaridade estratégica $(a=0)$.

Figura 1

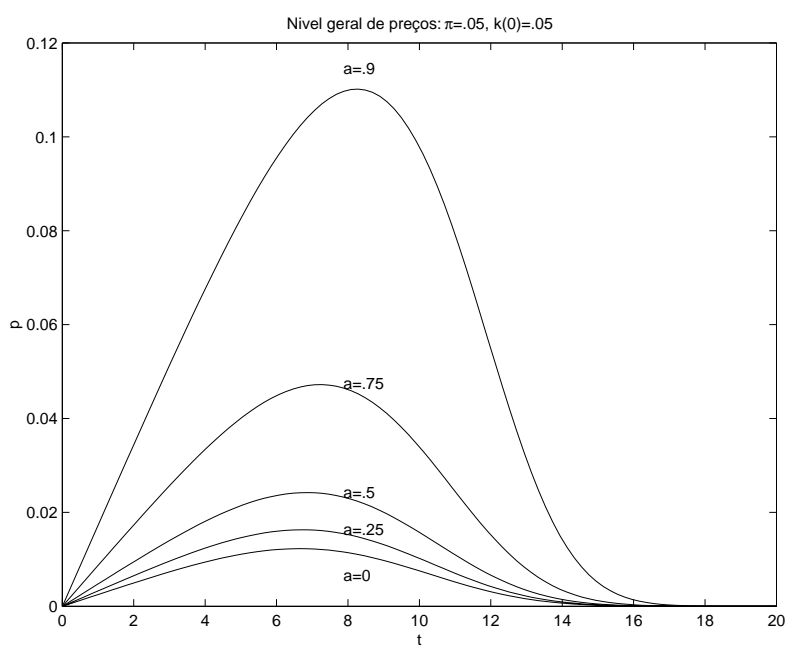

\footnotetext{
${ }^{9}$ Note, ainda, que a derivada do nível geral de preços é positiva para instantes de tempo superiores ao da efetivação da estabilização. Ao contrário, gerando inércia na taxa de inflação.
} 
Em primeiro lugar, o efeito da complementariedade estratégica sobre a magnitude do nível geral de preços (e como conseqüência, no produto) é bastante grande. O nível geral de preços atinge, em seu máximo, o valor (aproximado) de 0,012 para $a=0$ e 0,11 para $a=0,9$. Em outras palavras, os efeitos da passividade $(k(0)=0,05)$ são dez vezes maiores no caso em que há alta complementaridade estratégica, embora a persistência dos efeitos seja semelhante nos dois casos.

O resultado acima torna importante a questão da plausibilidade de um alto grau de complementaridade estratégica, o que nos remete aos determinantes do preço ótimo desejado por uma firma individual. Para análise desta questão observe que quando existe um aumento no nível geral de preços este causa dois efeitos no preço ótimo desejado. Por um lado, se não houvesse efeitos na demanda agregada, cada firma desejaria manter o mesmo preço relativo, aumentando portanto o seu preço proporcionalmente. Entretanto, o aumento no nível geral de preços representa uma redução na quantidade real de moeda, e portanto na demanda agregada, e este último efeito faz com que o preço relativo desejado seja mais baixo. A composição dos dois efeitos faz com que a firma deseje aumentar o seu preço, mas menos que proporcionalmente ao nível geral de preços. Quanto menor o efeito redutor advindo da demanda agregada, mais próximo de proporcional é o efeito, e portanto maior o grau de complementaridade estratégica. ${ }^{10}$ A evidência empírica aponta na direção da existência de um alto grau de complementaridade estratégica, o que torna os resultados obtidos relevantes.

Podemos, portanto dizer que o nosso modelo gera custos substanciais de desinflação. Observe que este resultado independe do grau de credibilidade da desinflação, o que é realista e contrasta com os resultados obtidos com modelos de rigidez nominal. Nestes, em condições de credibilidade perfeita, o custo de desinflação é muito pequeno tanto nos modelos em que as regras de preços dos agentes são dependentes do tempo (Ball, 1994), quanto nos modelos com regras dependentes do estado (Almeida e Bonomo, 2002).

Um outro parâmetro importante para os resultados é a proporção inicial de agentes míopes. A figura 2 ilustra o resultado de que quanto maior a proporção de agente míopes, maior deve ser a inércia inflacionária. Na conclusão deste artigo argumentamos que a proporção de agentes míopes deve ser tanto maior quanto mais longo e estável for um processo inflacionário. Como conseqüência deve ser

\footnotetext{
${ }^{10}$ De acordo com a versão simplificada do modelo Blanchard e Kiyotaki (1987), onde os consumidores são também produtores, o grau de complementaridade estratégica é tanto maior quanto maior for a elasticidade substituição entre os bens e quanto menos decrescentes forem os retornos do trabalho e/ou menor for a elasticidade da utilidade marginal do laser.
} 
mais fácil desinflar uma economia que teve um surto inflacionário recente do que uma cujo processo inflacionário seja crônico. Este resultado parece estar de acordo com a evidência internacional.

Figura 2

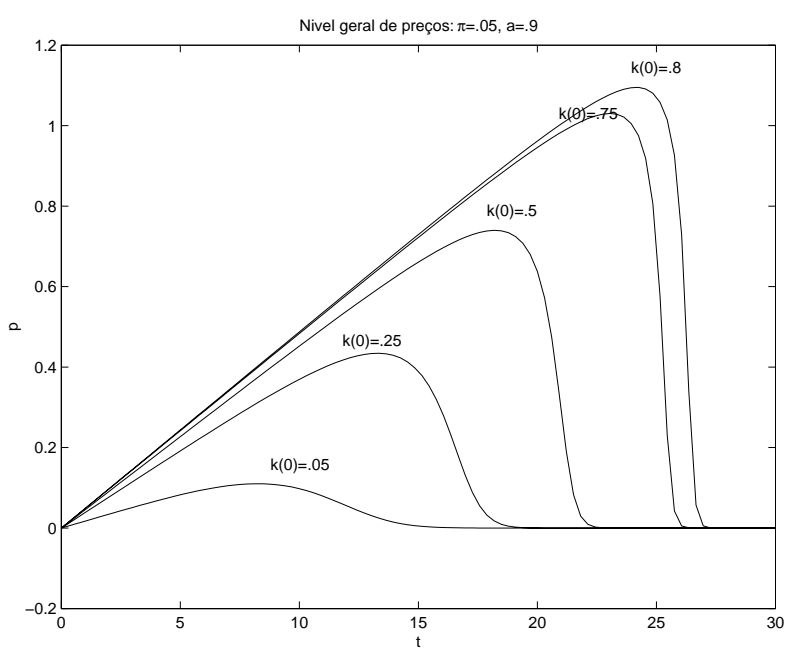

Outro resultado interessante gerado pelo nosso modelo é que a persistência do efeitos reais resultantes de um programa de estabilização são menores para taxas de inflação maiores (veja a figura 3). Claramente, isto decorre do fato de que a velocidade com que os agentes irracionais desaparecem da economia é maior quanto maior for $\pi$, como pode ser visto pela dinâmica (11'). Em outras palavras, nosso modelo prevê que os agentes conseguem se coordenar mais rapidamente em direção ao novo equilíbrio em situações de alta inflação. Como o nível de inflação residual gerado também é monotônico na inflação inicial, temos que desinflar uma economia com inflação mais alta gera uma recessão maior, porém mais curta. Este resultado é similar ao obtido por Bonomo e Carvalho (2003) para economias com rigidez nominal e regras de preço dependentes do tempo endogenamente determinadas. 
Figura 3

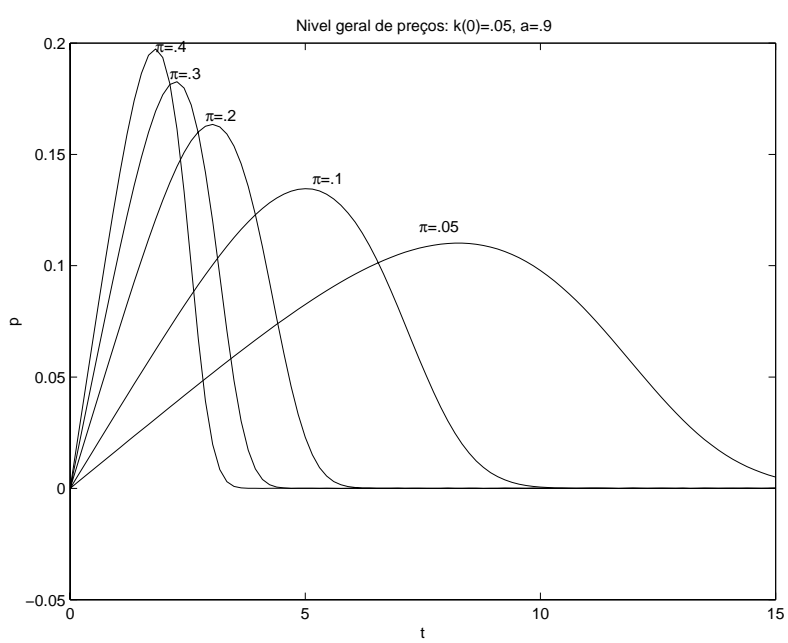

A seguir comparamos nossa formulação com as de Simonsen (1983), e Dow et alii (1993).

\subsection{Comparação com Simonsen (1983) e Dow et alii (1993)}

Simonsen (1983) supõe que os agentes estejam aptos a localizar o novo E.N. após a mudança de política monetária, mas que estejam incertos quanto ao fato dos outros agentes estarem jogando tal estratégia. Tal incerteza faz com que os agentes adotem estratégias maxmin que garantem ao menos a obtenção de um payoff mínimo no pior estado da natureza. ${ }^{11}$ Sob a hipótese de que os agentes adotam estratégias maxmin e supondo uma trajetória ( $a d$ hoc) para esta estratégia, Simonsen mostra que o resultado de estabilização indolor não mais vigora, gerandose inércia inflacionária e, dado o estoque nominal de moeda fixo, uma diminuição do estoque real de moeda.

Entretanto, ao mesmo tempo que parece-nos pouco razoável que os agentes estejam aptos a localizar imediatamente o novo E.N., isto é, que um equilíbrio de expectativas racionais vigore e que a redução da inflação à zero aconteça instantaneamente sem custo algum em termos de produto, tampouco parece-nos razoável

\footnotetext{
${ }^{11}$ Formalmente, uma estratégia maxmin resolve o seguinte problema: $\operatorname{Max}_{i} \operatorname{Min}_{j} U\left(a_{i}, a_{j}\right)$, onde $U(.,$.$) representa o payoff do jogador, a_{i}, i=1, \ldots, n$, representa o conjunto de ações do jogador e $a_{j}, j=1, \ldots, m$, representa o conjunto de ações a disposição do outro jogador.
} 
que os agentes sejam avessos ao risco ao extremo de forma a adotarem estratégias maxmin. No jogo de estabelecimento de preços com o qual estamos lidando, não há razão para que um jogador acredite que os outros jogadores ajam de forma a minimizar o seu payoff, pois não se trata de um jogo de soma zero. Em jogos não estritamente competitivos, a escolha da estratégia maxmin diante de uma probabilidade muito pequena de que o oponente, talvez por erro, escolha uma estratégia que minimize seu payoff é a grosso modo equivalente a uma aversão ao risco infinita.

Para gerar o seu resultado, Simonsen (1983) também precisa especificar, a cada momento, o conjunto de estratégias admissíveis que servirá de base para estratégia maxmin. A estratégia maxmin no seu modelo equivale a supor que os competidores escolham o preço máximo do conjunto de preços possíveis, onde este conjunto é definido de forma ad-hoc (por quê não de zero a infinito?). Para justificar o equilíbrio inflacionário inicial, este conjunto deve ser degenerado e incluir somente o preço de equilíbrio. Ele considera que quando há estabilização, o elemento máximo deste conjunto é o preço vigente anteriormente corrigido pela inflação passada. Consequentemente, a inércia inflacionária é gerada a partir da hipótese de que o elemento máximo do conjunto de preços possíveis aumenta a cada período de acordo com a inflação passada. Portanto, a inércia inflacionária do nível geral de preços é gerada a partir da hipótese de inércia inflacionária no preço máximo que uma firma pode cobrar.

Dow et alii (1993) dão uma formalização mais elegante à idéia de que uma aversão a perdas qualitativamente superior à implícita na maximização de utilidade esperada se constitui num ingrediente essencial do mecanismo de geração da inércia inflacionária. Utilizam-se da formalização de incerteza knightiana de Schmeidler e Gilboa (Schmeidler (1989), Gilboa (1987) e Gilboa e Schmeidler (1989)), e da sua posterior incorporação ao conceito de solução de jogos por Dow e Werlang (1994). Substituem a solução maxmin por E.N. com incerteza knightiana. Entretanto, a utilização deste conceito de solução exige que se defina o conjunto de estratégias possíveis para cada jogador. De novo, a imposição da hipótese de inércia inflacionária do preço máximo admissível para cada jogador gera a inércia da inflação do nível geral de preços.

A hipótese que adotamos nos parece mais razoável e menos artificial, embora esteja sujeita a outras críticas. Durante o equilíbrio inflacionário é plausível supor que muitos dos agentes ajustem seus preços automaticamente, sem racionalizar, pois esta forma de agir não tem muitos custos. Quando a situação muda, os agentes racionais recalculam as suas estratégias enquanto os agentes passivos vão deixando de sê-los na medida em que percebem as perdas de lucro geradas pela 
sua estratégia, vis a vis, seus competidores racionais. No nosso modelo, o que gera resultados é a inércia de comportamento de alguns agentes, que aprendem a partir de suas perdas. O grau de inércia gerado depende inversamente da velocidade de aprendizado. Portanto, os nossos resultados seguem da hipótese de que pelo menos um grupo de agentes não é tão racional quanto o suposto no modelo de expectativas racionais. Enquanto os agentes de Simonsen (1983) e Dow et alii (1993) diferem dos agentes do modelo convencional por serem qualitativamente mais avessos a perdas.

Por outro lado a nossa modelagem não é um problema de coordenação clássico, no sentido de que todos os agentes estão piores somente porque não sabem como os outros vão agir, pois os passivos agem da mesma forma por inércia e os ativos agem sabendo como os passivos vão agir. Entretanto, nosso problema pode ser considerado um problema de coordenação porque os agentes passivos não preferem a estratégia que adotam e todos os agentes estariam melhor caso os agentes passivos adotassem a nova estratégia de equilíbrio. Neste sentido um mecanismo de coordenação que implementasse o novo equilíbrio seria igualmente eficaz em ambas as situações.

\section{Conclusão}

Na segunda seção, apresentamos o modelo em que a autoridade monetária anuncia e efetiva o programa de estabilização de preços (credibilidade perfeita). Mostramos que a existência de uma pequena proporção de agentes passivos em conjunção com complementaridade estratégica pode gerar substancial custos para a estabilização.

Um resultado interessante é que o modelo prevê menor persistência dos efeitos reais em situações de alta inflação, pois a taxa de desaparecimento dos agentes passivos é maior em situações de alta inflação inicial. Em outras palavras, nosso modelo sugere que os agentes se coordenam mais rapidamente para o novo equilíbrio em situações de alta inflação. Este resultado é similar ao obtido por Bonomo e Carvalho (2003), num modelo com rigidez nominal e regras de preços endógenas dependentes do tempo. Blanchard (1997) sugere que é mais fácil desinflar a partir de inflações altas devido ao menor grau de rigidez nominal. Nosso modelo sugere que a recessão é mais curta, porém mais profunda em uma situação em que a desinflação é totalmente crível. Os resultados deste trabalho são estendidos em Bonomo et alii (2003) para desinflações lineares, com possibilidade de retrocesso por parte da autoridade monetária.

Uma limitação do nosso trabalho é a exogeneidade da proporção de agentes 
passivos. Uma extensão possível seria tornar esta proporção endógena, de acordo com a seguinte sugestão. Formular-se-ia uma dinâmica para a proporção de agentes passivos durante o estado estacionário. Para isto impor-se-ia um pequeno custo adicional (por período) em ser ativo, que representaria o custo de calcular a estratégia ótima continuamente. Num estado estacionário o número de agentes passivos deveria crescer, pois afora o custo de ser ativo o payoff seria o mesmo para ambos os agentes. Assim quanto mais a economia permanecesse num estado estacionário, maior o número de agentes passivos. De acordo com o nosso modelo, durante transições a proporção de agentes passivos decresce. O modelo teria que ser fechado com uma dinâmica que determinasse a persistência de estados estacionários.

\section{Referências}

Akerloff, G. \& Yellen, J. L. (1985a). Can small deviation from rationality make significant differences to economic equilibria? American Economic Review, 75:708-721.

Akerloff, G. \& Yellen, J. L. (1985b). A near-rational model of the business cycle with wage and price inertia. Quarterly Journal of Economics, 100:823-838.

Almeida, H. \& Bonomo, M. (2002). Optimal state-dependent rules, credibility, and inflation inertia. Journal of Monetary Economics, 49:1317-1336.

Amato, J. D. \& Laubach, T. (2003). Rule of thumb behavior and monetary policy. European Economic Review, 47:761-790.

Ball, L. (1991). The genesis of inflation and the costs of disisinflation. Journal of Money, Credit and Banking, 23:439-452.

Ball, L. (1994). Credible disinflation with staggered price setting. American Economic Review, 84:282-290.

Ball, L. (2000). Near rationality in a two monetary regimes. NBER Working Paper 7988.

Blanchard, O. (1997). Comment on "stopping hyperinflations, big and small", by Peter Ireland. Journal of Money, Credit and Banking, 29:776-782.

Blanchard, O. \& Kiyotaki, N. (1987). Monopolistic competition and the effects of agregate demand. American Economic Review, 77:647-666. 
Bonomo, M. (1992). Dynamic pricing models. Chapter 1, Ph.D. Dissertation, Princeton University.

Bonomo, M., Carrasco, V., \& Moreira, H. (2003). Aprendizado evolucionário e custo médio da desinflação. EPGE, mimeo.

Bonomo, M. \& Carvalho, C. (2003). Endogenous time-dependent rules, and inflation inertia. Journal of Money, Credit and Banking. No prelo.

Cooper, R. \& John, A. (1988). Coordinating coordination failures in keynesian models. Quarterly Journal of Economics, 103:441-463.

Dow, J., Simonsen, M. H., \& Werlang, S. (1993). Knightian rational expectations, inflationary inertia and money neutrality. mimeo, EPGE/FGV.

Dow, J. \& Werlang, S. (1994). Nash equilibrium under knightian uncertainty: Breaking down backward induction. Journal of Economic Theory, 64(2):305324.

Fudenberg, D. \& Levine, D. (1998). The Theory of Learning in Games. M.I.T. Press, Cambridge.

Gilboa, I. (1987). Expected utility with purely subjective non-additive priors. Journal of Mathematical Economics, 16:279-304.

Gilboa, I. \& Schmeidler, D. (1989). Maxmin expected utility with a non-unique prior. Journal of Mathematical Economics, 18:141-153.

Lucas, R. (1986). Adaptive behavior and economic theory. Journal of Business, 59:401-425.

Roberts, J. (1997). Is inflation sticky? Journal of Monetary Economics, 59:173196.

Schmeidler, D. (1989). Subjective probability and expected utility without additivity. Econometrica, 57:571-587.

Simonsen, M. H. (1983). Price stabilization and income policies: Theory and the brazilian case study. In Dornbusch, Rudiger \& Simonsen, M. H., editor, Inflation, Debt and Indexation. MIT Press, Cambridge.

Weibull, J. W. (1996). Evolutionary Game Theory. MIT Press, Cambridge. 